\title{
Automated segmentation of the atrial region and fossa ovalis towards computer-aided planning of inter-atrial wall interventions
}

\author{
Pedro Morais ${ }^{\mathrm{a}, \mathrm{b}, \mathrm{c}, \mathrm{d}}$, João L. Vilaçaa ${ }^{\mathrm{a}, \mathrm{b}, \mathrm{e}}$, Sandro Queirós ${ }^{\mathrm{a}, \mathrm{b}, \mathrm{d}, \mathrm{f}}$, Alberto Marchi $^{\mathrm{g}}$, Felix Bourier ${ }^{\mathrm{h}}$, \\ Isabel Deisenhofer ${ }^{\mathrm{h}}$, Jan D’hooge ${ }^{\mathrm{d}}$, João Manuel R.S. Tavares ${ }^{\mathrm{c}, *}$ \\ ${ }^{a}$ Life and Health Sciences Research Institute (ICVS), School of Medicine, University of Minho, 4710-057 Braga, Portugal \\ ' ICVS/3B's - PT Government Associate Laboratory, Braga/Guimarães, Portugal \\ ${ }^{\mathrm{C}}$ Instituto de Ciência e Inovação em Engenharia Mecânica e Engenharia Industrial, Faculdade de Engenharia, Universidade do Porto, Portugal \\ ${ }^{\mathrm{d}}$ Lab on Cardiovascular Imaging \& Dynamics, Department of Cardiovascular Sciences, KULeuven - University of Leuven, Leuven, Belgium \\ e $2 A i$ - Polytechnic Institute of Cávado and Ave, Barcelos, Portugal \\ ${ }^{\mathrm{f}}$ Algoritmi Center, School of Engineering, University of Minho, Guimarães, Portugal \\ g Cardiomyopathies Unit, Careggi University Hospital Florence, Italy \\ h German Heart Center Munich, Technical University, Munich, Germany
}

\section{A R T I C L E I N F O}

\section{Article history:}

Received 21 February 2018

Revised 29 March 2018

Accepted 17 April 2018

\section{Keywords:}

Image segmentation

Cardiac intervention planning

Inter-atrial wall interventions

Competitive segmentation strategy

Atlas-based initialization

\begin{abstract}
A B S T R A C T
Background and objective: Image-fusion strategies have been applied to improve inter-atrial septal (IAS) wall minimally-invasive interventions. Hereto, several landmarks are initially identified on richly-detailed datasets throughout the planning stage and then combined with intra-operative images, enhancing the relevant structures and easing the procedure. Nevertheless, such planning is still performed manually, which is time-consuming and not necessarily reproducible, hampering its regular application. In this article, we present a novel automatic strategy to segment the atrial region (left/right atrium and aortic tract) and the fossa ovalis (FO).

Methods: The method starts by initializing multiple 3D contours based on an atlas-based approach with global transforms only and refining them to the desired anatomy using a competitive segmentation strategy. The obtained contours are then applied to estimate the FO by evaluating both IAS wall thickness and the expected FO spatial location.

Results: The proposed method was evaluated in 41 computed tomography datasets, by comparing the atrial region segmentation and FO estimation results against manually delineated contours. The automatic segmentation method presented a performance similar to the state-of-the-art techniques and a high feasibility, failing only in the segmentation of one aortic tract and of one right atrium. The FO estimation method presented an acceptable result in all the patients with a performance comparable to the inter-observer variability. Moreover, it was faster and fully user-interaction free.

Conclusions: Hence, the proposed method proved to be feasible to automatically segment the anatomical models for the planning of IAS wall interventions, making it exceptionally attractive for use in the clinical practice.
\end{abstract}

(C) 2018 Elsevier B.V. All rights reserved.

\section{Introduction}

Anatomically, the atria are complex cardiac chambers with high variability between subjects [1,2]. Although their body is typically described as simple concave shapes in normal cases [3], atrial enlargement of one chamber is common, negatively affecting the

\footnotetext{
* Corresponding author.

E-mail addresses: pedromorais@med.uminho.pt (P. Morais), jvilaca@ipca.pt (J.L. Vilaça), sandroqueiros@med.uminho.pt (S. Queirós), fbourier@googlemail.com (F. Bourier), deisenhofer@dhm.mhn.de (I. Deisenhofer), jan.dhooge@uzleuven.be (J. D’hooge), tavares@fe.up.pt (J.M.R.S. Tavares).
}

other chamber and changing their anatomies [4]. Moreover, high spatial and shape variations are frequently found in their connected structures (i.e. pulmonary veins - PV, vena cava -VC and appendages) and even in specific anatomical regions (e.g. fossa ovalis - FO) $[1-3,5]$. Due to all these reasons, minimally invasive atrial interventions are difficult, requiring multiple imaging modalities for planning (mainly computed tomography - CT) and guidance (e.g. ultrasound) [6,7]. Firstly, during the planning stage, the clinician visually evaluates the richly detailed images, searching for anatomical variations. Next, throughout the intervention, real-time images are used to guide the different instruments inside the human body until the target positions [7]. 
Traditionally, both planning and interventional data are independently evaluated in a manual manner, being subsequently mentally combined by the expert throughout the intervention [7]. Nevertheless, such approach requires great experience, is very timeconsuming, and frequently results in procedural failures and complications $[7,8]$. Thus, automated image-fusion strategies were proposed $[9,10]$. Usually, these strategies start with a segmentation of the target structures, being subsequently fused with the intraprocedural data [9]. As a result, the high-detail of the pre-operative stage is transferred to the intervention, enhancing and improving intra-procedural images $[7,9]$.

The success of the aforementioned image-fusion strategies is directly related to the accuracy of the image alignment approach [7]. While some researchers focused on strategies to align the preand intra-procedural data using 2D/3D alignment approaches [10], landmark identification [11] and/or image registration [12], other researchers optimized the planning stages suggesting automated algorithm versions [2,13]. These automated methods showed a performance comparable to the manual analysis [2,14,15], requiring none or little user input, removing or minimizing the variability between observers and reducing the time spent during the planning [2]. Specifically for cardiac applications, atlas-based $[16,17]$, deformable models $[14,18]$ and machine learning strategies [15] were proposed to segment the different cardiac chambers. Particularly, the methodologies described by Ecabert et al. [14] and Zheng et al. [15] successfully segmented the four cardiac chambers plus the attached great vessels, and the atlas-based methodology from Kirisli et al. [16] proved its added-value for cardiac chamber segmentation in large databases of contrast-enhanced [16] and non-contrast-enhanced CT images [19]. However, these solutions still present inaccurate results in the thin mid-walls, showing overlapping regions [16] or merging of different contours [14]. As such, we recently proposed a competitive deformable model strategy to segment the atrial region (i.e. atrial bodies and aortic tract - AO) with a correct delineation of the mid atrial walls [18]. Such formulation allowed correct evaluation of the inter-atrial septal (IAS) wall, allowing its application for IAS interventions (e.g. transseptal puncture or atrial septal defect closure) and even improving the planning of multiple minimally invasive atrial interventions (e.g. atrial fibrillation, atrial appendage closure) [5,20]. However, a semi-automatic version was described in [18], requiring an initialization of each chamber. Moreover, specific landmarks/regions inside the inter-atrial wall, such as FO [5], were not detected.

The fossa ovalis is the thinnest region of the IAS wall, presenting an oval or circular shape and usually located posteriorly, at the junction of the mid and lower third of the right atrium (RA) [6]. It is described as a depression, composed by a thin flap (floor of the FO) and its surrounding margins designated as FO limbus [21]. Since direct physical access to the left atrium (LA) is not possible, the FO is used as an access point between the RA and LA $[5,6]$. This technique is termed transseptal puncture, which consists of a needle that is inserted percutaneously until the RA, puncturing the FO and accessing the LA [6]. The correct identification of the FO is crucial to identify the optimal access route between both atria [11], preventing complications, or multiple puncture attempts [5]. Moreover, the FO identification is also relevant in other LA interventions, namely for device selection and optimal route identification for catheter ablation, mitral valve (MV) replacement and left atrial appendage (LAA) occlusion [5,20,21]. Some researchers have investigated the added-value of the FO identification in a preinterventional stage, proving that it eases the real intervention, especially for challenging anatomies $[9,22,23]$. Nonetheless, its identification is still performed manually [9,22], hampering its application in the clinical practice.

To overcome the aforementioned difficulties, a fully automatic segmentation strategy is proposed in this article to generate the

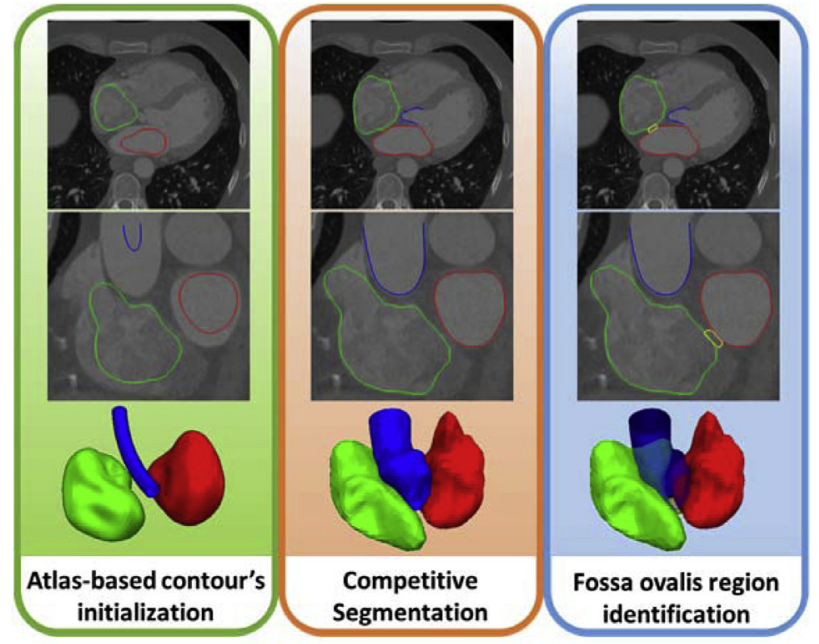

Fig. 1. Overview of the proposed fully automatic method for atrial region segmentation (red: LA, green: RA, blue: AO) and fossa ovalis identification (yellow). (For interpretation of the references to colour in this figure legend, the reader is referred to the web version of this article.)

atrial region models and the FO in CT datasets. Both models can improve the current IAS interventional planning, making it faster, more reproducible and simpler. The method starts by segmenting the relevant cardiac chambers (i.e. atrial region) around the IAS wall, allowing an accurate evaluation of wall thickness and shape. The proposed segmentation method is an extension of [18], with a robust initialization through an atlas-based technique. Then, since a correct representation of the IAS wall is used, the FO region is identified by assessing the intermediate mid wall positions of all contour pairs and spatial location information.

Overall, this work introduces three novelties: 1) extension of our previously presented atrial segmentation method with a fully automatic one; 2 ) a novel methodology to accurately segment the FO; 3 ) a clinical validation of the proposed method.

This article is structured as follows. In Section 2, a technical description of the proposed fully automatic atrial segmentation method is presented. In Section 3, the validation experiments and their results are presented. Section 4 discusses the performance of the proposed method against the state-of-the-art and expected inter-/intra-observer variability. Finally, the conclusions of this study are presented in Section 5.

\section{Methods}

The proposed fully automatic method comprises three sequential conceptual blocks (Fig. 1): 1) automatic and rough identification of the cardiac chambers through an atlas-based technique with global transformation models; 2) refinement of the target region boundaries using a competitive segmentation methodology that guarantees the integrity of the mid thin walls; and 3) identification of the FO region through the combination of the patientspecific wall thickness information (extracted from the anatomical models obtained in (2)) and a set of known anatomical references that provides the spatial location.

\subsection{Atlas based contour's initialization}

Due to the high feasibility previously demonstrated by atlasbased techniques [16], a similar methodology was selected to initialize our method. The traditional formulation finds the optimal transformation that aligns the target image with a labelled dataset (designated as atlas), subsequently applying it to the labels and 


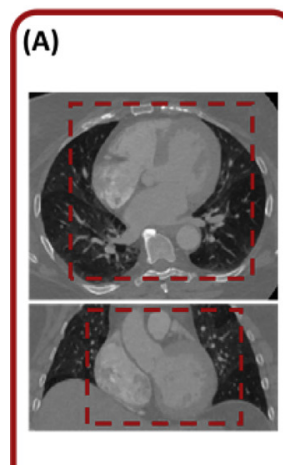

Pre-processing

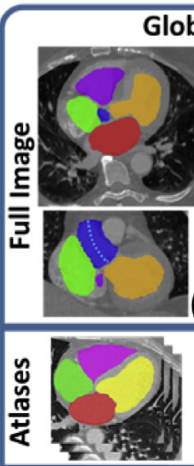
and

Atlas alignment

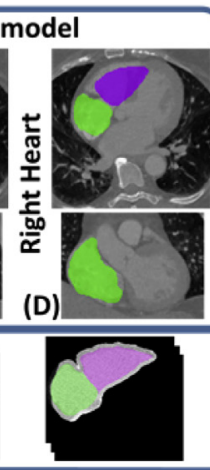

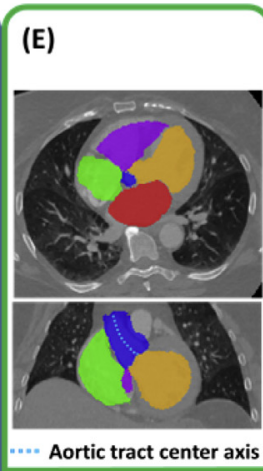

Label fusion

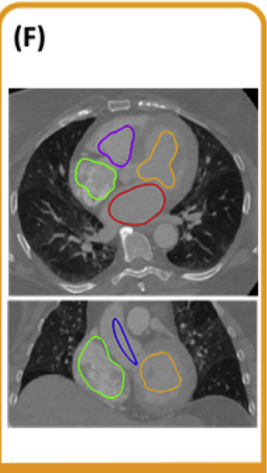

Post-processing

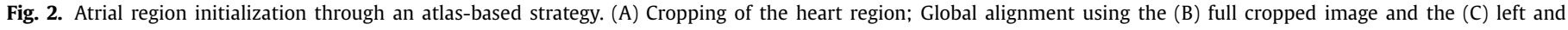
(D) right heart. (E) Label fusion using a majority voting and final post-processing of each label (F).

transferring the relevant anatomies to the target image. Different transformation models have been used, for example, global transforms (rigid or affine) are used to roughly align the different cardiac cavities and local transforms (deformable registration) are then applied to adapt the results toward the anatomy [16,17]. Since anatomical variations are expected, multiple atlases are usually used and all the results are combined through a label fusion process. However, the current strategies still present disadvantages: they are very time-consuming, the resulting shape variability is dependent of the atlases used, the target and atlas image should present a similar field of view (FOV) and should be in the same cardiac phase $[2,16,17]$.

Taken into consideration all the aforementioned drawbacks, we modified the traditional atlas-based approach [16] through:

1 - A pre-processing strategy that defines a FOV around the heart region (Fig. 2A). The method defines a bounding box using the lung positions, which are binarized through the Hounsfield units (HU). The inferior limit of the bounding box is defined by the inferior tip of the lungs and its central position is defined by the centroid of both binarized lungs. Of note, the lungs were selected, since they are always visible on cardiac CT images and due to its particular HU value.

2 - Instead of global and local registrations, the current strategy only applies affine transforms, allowing a fast initialization of the cardiac anatomy. Moreover, a two-stage sequential process is used: i) a full image and ii) a regional (left or right heart) alignment. The full image approach aligns the entire image (target image and atlas), initializing all chambers simultaneously (Fig. 2B). The resulting contours are then applied to mask the left (Fig. 2C) and right heart (Fig. 2D) in the target image. In order to prevent overlaps between the left and right alignment stages and to compensate sub-optimal global alignments, each masked region is dilated (in our experiments, by $10 \mathrm{~mm}$ ). Finally, both regions are independently aligned with masked left and right atlases, respectively.

3 - The AO center axis (defined as 5 points) is also transferred to the target image (dashed line in Fig. 2) using the optimal transformation. The axis is later referred to in Section 2.2.1.

4 - The final label fusion is performed for all organs at once using a majority voting [24] (Fig. 2E). Next, a post-processing based on Otsu threshold is applied [25] (Fig. 2F), improving the robustness of the method for sub-optimal results. In detail, the preliminary result of each chamber is used to mask the image, and each masked region is then divided into two groups based on its image intensities. The group with the largest number of voxels is selected as the final result. Specifically for the AO, we fit a spline to all possible points obtained previously.

5 - Two atlas database are used: i) a public database [16] with 8 atlas at end-diastole (ED), and ii) an in-house database with 4 atlas at end-systole (ES). The phase of the target image should be selected or extracted from the acquisition parameters.

\subsection{Competitive segmentation}

The proposed segmentation method relies on our previously developed atrial region segmentation strategy [18]. A set of independent functions are used to control the evolution of each $3 \mathrm{D}$ contour (i.e. LA, RA, AO, left ventricle - LV, right ventricle - RV), and a set of image descriptors are used to identify the optimal boundaries. The segmentation problem is an iterative process guided by intensity-based (signed local Yezzi - SLY, [26]) and edge-based energies (unsigned distance function to the nearest edge). The edge map is generated using a Canny edge filter. In order to prevent overlapping regions, a competitive approach is applied between all the contours pairs. In detail, when the distance between two contours is lower than a pre-defined value $\left(R_{T}\right)$, a force with opposite direction to the propagation direction is applied to each contour, locally preventing fast steps or even changing the contour evolution direction. The magnitude of the competitive force is computed based on the distance between contours, assuming a small value when the distance between contours is near $R_{T}$ (allowing refinement to mid walls) and applying a high penalization (preventing overlapping) when the distance is almost zero.

In order to make the method computationally attractive, the Bspline Explicit Active Surfaces (BEAS) framework is used [27]. As such, each contour uses an analytic representation described as a linear combination of B-splines. Furthermore, explicit functions (cylindrical model for the AO and spherical representation for the remaining contours), and independent smoothing terms are applied to each contour, reducing the dimensionality of the segmentation problem and allowing independent control of the smoothing degree of each contour, respectively.

The segmentation pipeline relies on [18], namely: 1) fast growing of the initialized contours using a competitive BEAS (CBEAS) threshold with global descriptors (Fig. 3A); 2) AO axis correction (Fig. 3B); 3) contour refinement using the CBEAS segmentation with local descriptors (Fig. 3C). Note that the LV and RV contours are only evolved throughout the first stage to define the valve planes. Moreover, contrary to [18], an axis correction stage (Section 2.2.1) is now included to compensate for sub-optimal AO axis results throughout the Section 2.1 . 


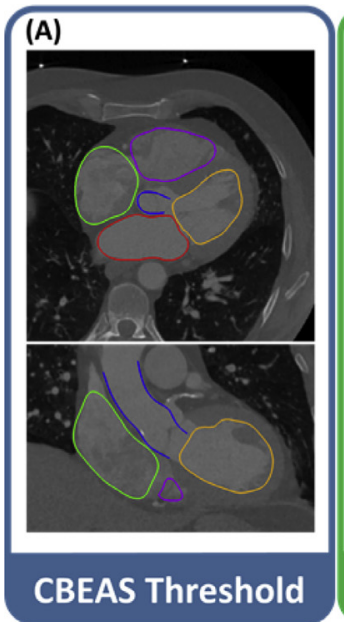

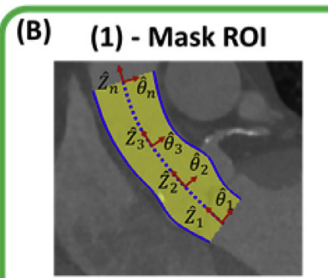

(3) - Leaflets identification

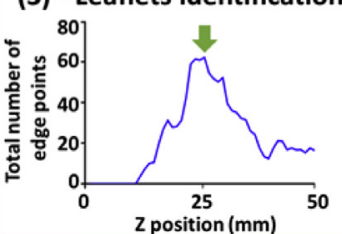

Aortic axis correction

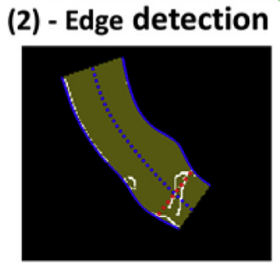

(4) - Axis correction

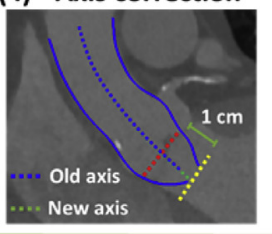

.

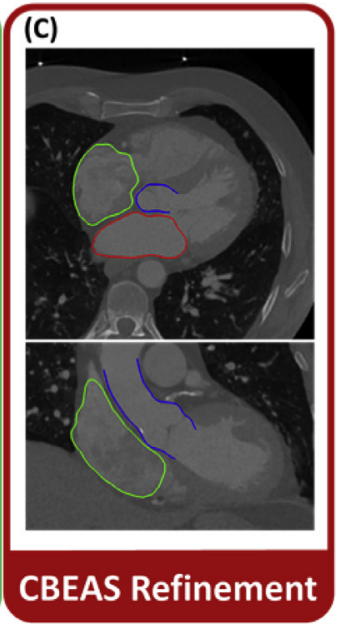

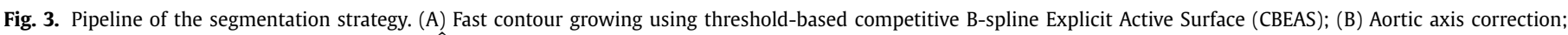
and $\mathrm{C}$ ) Contours refinement using local CBEAS. $\hat{\theta}$ and $\hat{z}$ represents the coordinates of the cylindrical space.

As the output of this step, a set of meshes $S_{i}$ with $i \in\{L A, R A$, $A o\}$ are obtained. Each mesh is represented by multiple vertices $\boldsymbol{x}=[x, y, z]$ and a connectivity matrix $\boldsymbol{c}$.

\subsubsection{Aortic axis correction}

The atlas-based technique allows a coarse identification of the $\mathrm{AO}$ axis, presenting a sub-optimal performance in the left ventricular outflow tract (LVOT) region, due to the lack of walls. Inspired by [13], we propose an AO axis correction technique. The method starts by estimating the leaflets positions through a Canny edge filter (Fig. 3B-2) applied on a region-of-interest (ROI). The ROI is defined based on the $\mathrm{AO}$ contour computed at the BEAS-threshold stage (Fig. 3B-2). Then, the obtained edge map is represented in the cylindrical space. For each $\hat{z}$ level, the number of $\hat{\theta}$ coordinates with an edge point is counted, and the level with the maximum number of edges is used to define the leaflets regions (Fig. 3B3 ). Finally, we generate a novel axis assuming a fixed spacing (in our results this spacing was defined equal to $10 \mathrm{~mm}$ ) between the leaflets and the LVOT plane (Fig. 3B-4), and reinitialize the AO contour.

\subsection{Fossa ovalis identification}

The FO is identified by the evaluation of two different parameters (Fig. 4): i) IAS wall thickness (Section 2.3.1), computed using the boundary of the atrial contours; and ii) spatial location of the expected FO region (Section 2.3.2), aligning the obtained anatomical atrial surfaces with manual delineated references that contain the FO region. While the first parameter provides accurate anatomical information, the second one computes a smooth voting map that represents the FO spatial variation. Thus, by combining both parameters, local minima caused by incorrect segmented regions or incorrect spatial locations are penalized, enhancing the real FO region.

In order to simplify the searching of the FO region, the proposed method is applied to the LA surface only. In this sense, the FO at the left heart is initially located, and then interpolated towards a $3 \mathrm{D}$ volume using the spatial relation between chambers. It might be noted that the LA contour is used due to its superior image quality and higher segmentation accuracy [18].

\subsubsection{Inter-atrial septal wall thickness estimation}

In order to estimate the IAS wall thickness, an extra segmentation process is applied focused on the mid walls. Although this information could be extracted from atrial contours, its result is dependent on the segmented anatomical regions in all chambers. Since the FO region is located near the intersection between the inferior vena cava (IVC) and the RA body (when evaluated from the right heart), its accurate detection is hampered due to a suboptimal RA segmentation result in the IVC. In this sense, a new contour focused on the particularities of the IAS wall shape is initially and evolved (Fig. 4B). This contour is defined based on the LA position and its evolution is controlled by the previously obtained atrial models (Fig. 4A). Thus, the wall details found between the atrial contours are directly integrated on the final IAS surface, and also applied to accurately estimate the wall thickness in regions with sub-optimal segmentation result.

Starting from the LA contour, an extra BEAS surface representing the wall thickness is initialized (Fig. 4B). Both surfaces are combined using a coupled strategy (Fig. 4C), meaning that the wall surface is represented based on the LA contour position $\left(\psi^{\text {Pos }}\right)$ plus the wall thickness $\left(\psi^{\text {Thick }}\right)$. The reader is kindly directed to supplementary material I and for [28] for further details on coupled methodologies in the BEAS framework.

The original LA contour is not updated throughout this stage, working only as an anchor for the segmentation of the mid-walls and keeping the $\psi^{\text {Pos }}$ unaltered. As such, a novel optimization process targeting the identification of the optimal thickness map $\psi^{\text {Thick }}$ is started. Specifically, the evolution of the IAS contour is guided by a SLY energy (targeting black to white transitions) with no edge terms. Negative thicknesses are not allowed, preventing overlaps between both LA and IAS contours [26].

In order to ease the wall boundaries identification, a competitive strategy is also applied between the IAS contour and the RA and AO contours. Again, the RA and AO are not optimized during this stage, working as a barrier. Since no empty spaces are physically expected between the IAS wall and the AO|RA, the $R_{T}$ was set to $0 \mathrm{~mm}$, penalizing overlapping regions only.

The wall thickness map $I A S_{\text {Thick }}(\boldsymbol{x})$ is finally defined at the vertices $\boldsymbol{x}$ of the LA contour and computed as the distance between $S_{L A}$ and the IAS wall contours (Fig. 4C). Finally, relative distances are computed by normalizing the value in each vertex by the maximum value in the map.

\subsubsection{Fossa ovalis spatial alignment}

The FO spatial location is estimated based on a surface alignment technique applied between the automatic and a set of references/templates with the desired anatomy. The references correspond to datasets manually evaluated by one expert, which in- 


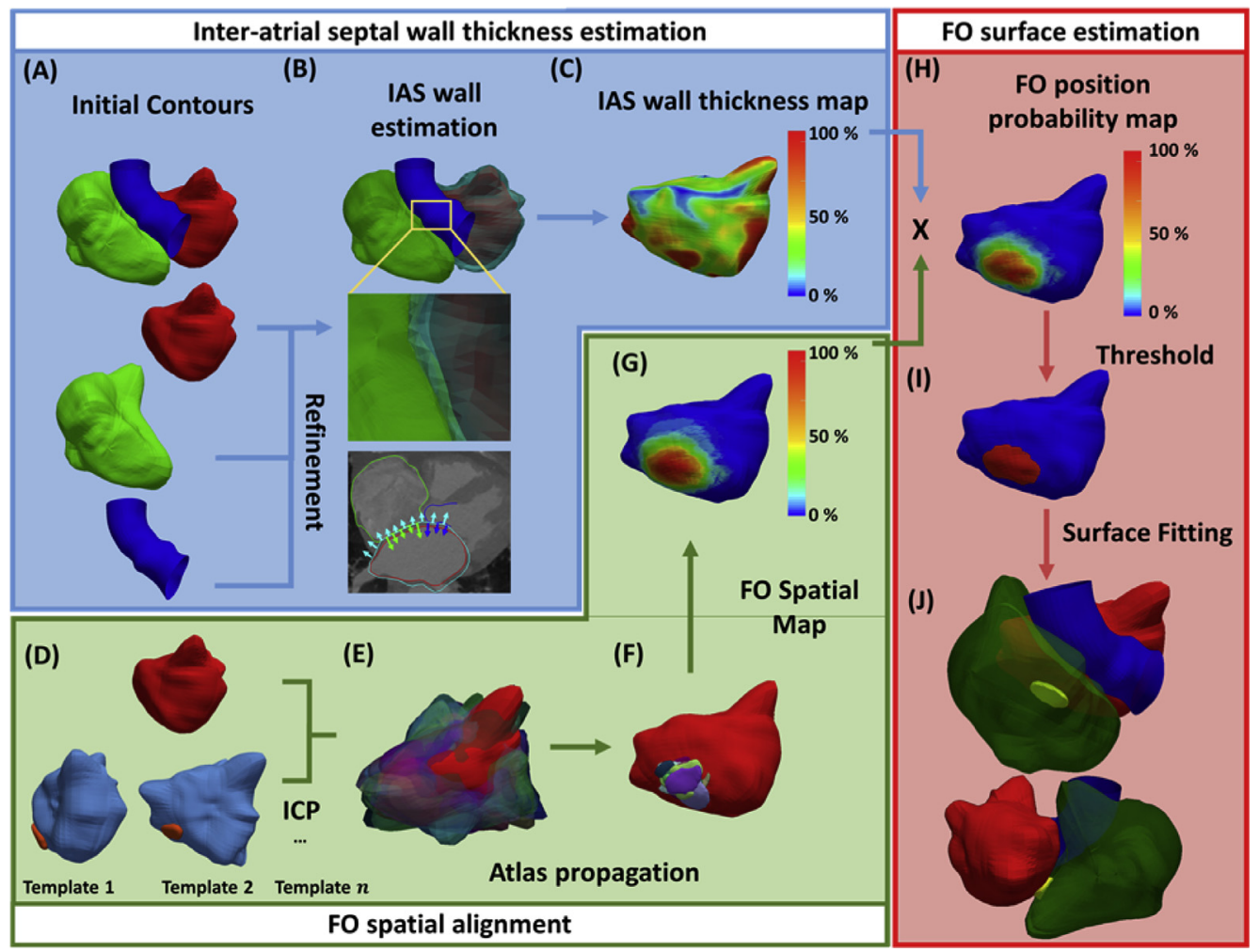

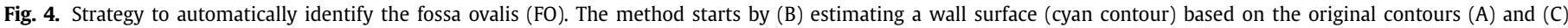

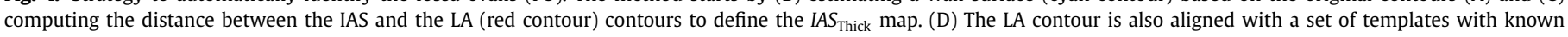

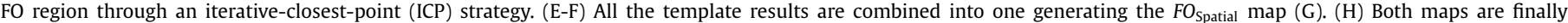

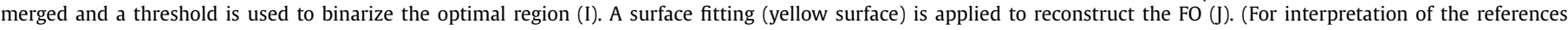
to colour in this figure legend, the reader is referred to the web version of this article.)

cludes both LA and FO surfaces (Fig. 4D). In detail, the target LA chamber is aligned with a set of LA references using an iterative closest point (ICP) strategy (Fig. 4E). We start by rigidly aligning both surfaces and then compensating the scaling differences using a similarity transform. The final transform for each case is independently applied to the FO label, propagating it to the target LA (Fig. 4F). Then, the distance between each point of the transformed FO surface and the vertices $\boldsymbol{x}$ of the automatic LA is computed, and the nearest LA vertices considered as valid FO points.

Finally, a voting map $\mathrm{FO}_{\text {Spatial }}(\boldsymbol{x})$ is constructed, by counting the different number of FO templates in each vertices $\boldsymbol{x}$ and normalizing it with the maximum value (Fig. 4G).

\subsubsection{Fossa ovalis surface estimation}

FO probability map estimation and binarization: The final probability map is obtained by combining both $I A S_{\text {Thick }}$ and $\mathrm{FO}_{\text {Spatial }}$ maps in each vertices $\boldsymbol{x}$ through (Fig. 4H):

$F O_{\text {Position }}(\boldsymbol{x})=\left(1-I A S_{\text {Thick }}(\boldsymbol{x})\right) \times F O_{\text {Spatial }}(\boldsymbol{x})$.

Both $I A S_{\text {Thick }}$ and $F O_{\text {Spatial }}$ are normalized between [0-1], with 1 representing the maximal thickness position and the optimal anatomical location of FO, respectively. By inverting the $I A S_{\text {Thick }}$ map and multiplying it with the $\mathrm{FO}_{\text {Spatial }}$, the FO region is enhanced.

Finally, the optimal position of the FO is extracted from the

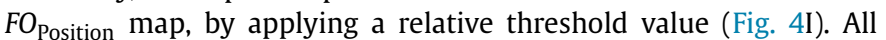
the positions $\boldsymbol{x}$ with a value higher than this threshold are considered as valid FO points.

FO surface fitting: Starting from the valid FO points, a 3D FO surface is reconstructed based on the spatial relations between the chambers. As such, we compute the distance between each FO point and the remaining surfaces (i.e. RA and AO). In detail, for each selected point, the nearest position is selected as a valid FO boundary, generating a point cloud representing the FO anatomy. Finally, a structured surface is estimated by deforming an ideal spherical mesh with the point-cloud through [29] (Fig. 4J).

\section{Experiments}

The new fully automatic framework (henceforward referred as FA) was evaluated based on: 1) the differences found between the FA atrial region segmentation strategy relatively to the previous validated semi-automatic technique (termed SA) and manual contours; and 2) the analysis of the FO estimated by the proposed strategy and the corresponding one manually delineated.

\subsection{Data description}

Forty-one datasets retrospectively obtained in clinical practice from patients with suspicion of pathology in the atria were used. In detail, patients with normal anatomy, pacemaker, prosthetic valves, atrial body enlargement and candidates to pulmonary vein ablation were included. ECG-gated cardiac multi-slice CT images were acquired with a multi-detector SOMATOM Force (Siemens, Germany) CT scanner, with the number of rows varying between 64-128 slices. An image resolution of $0.69 \times 0.69 \mathrm{~mm}^{2}$, slice thickness of $0.8 \mathrm{~mm}$, and a matrix size of $512 \times 512$ was used. The Xray tube current varied between $1844-2597 \mathrm{~mA}$ and the peak voltage varied between $70-110 \mathrm{kV}$. A contrast injection (Imeron 350 , Bracco-Byk Gulden, Konstanz, Germany) of 40-100 ml was applied and a unique phase was acquired (both end-diastolic and endsystolic phases were included). Each dataset was reconstructed using the convolution kernel Bv36d. All the described acquisition parameters were defined based on the normal clinical practice at the 
German Heart Centre Hospital. An overview of the clinical dataset cases can be found in Supplementary Material II.

\subsection{Fully automatic atrial region segmentation}

Ground truth generation: Both LA and RA contours were manually delineated by one expert using the semi-automatic CARTO3 segmentation tool (Biosense Webster, Diamond Bar, CA, USA), followed by manual corrections (as described in detail in [9]). The AO was manually delineated using an in-house software through the following steps: 1) AO centerline definition, by providing multiple clicks along the AO tract; 2 ) delineation of multiple planes (25-30 planes) perpendicular to the centerline and, 3) surface reconstruction. Moreover, similarly to our initial work, multiple bounding boxes were manually generated using a $3 \mathrm{D}$ visualization software (Paraview Kitware Inc., USA [30]), limiting the influence of the connecting structures of the atrial body, i.e., PV, VC and left/right atrial appendages (LAA/RAA), in the final result of the proposed method. Note that each bounding box was generated around the ostia of each structure. Moreover, two planes representing the mitral and tricuspid valve (TV) were built and used to evaluate the accuracy of the proposed atrial body segmentation method. In detail, each plane was independently defined using the following strategy: 1) manual delineation of the valve's ring in several rotated planes (centered on the valve center position); and 2) plane fitting to these points.

Implementation details: The regular bounding box used to define the FOV in CT images (Section 2.1) had a size of $20 \times 20 \mathrm{~cm}$ along the axial direction, and its height was computed as the distance between the lung's centroid and the lung's tip. Regarding the segmentation parameters (Section 2.2), all were kept from the original work [18]. The reader is kindly directed to [18] for further details on them.

Statistical analysis: The FA contours were compared against the corresponding manual and SA results (available in [18]) in terms of absolute point-to-surface (P2S) distance, Dice coefficient (DC) and 95th percentile of the Hausdorff distance. Moreover, the influence of the generated bounding boxes and valve planes to separate the atrial body from its connected structures was also evaluated. Regarding the FA results, independent databases were used to initialize the method through the abovementioned atlas-based strategy (Section 2.1). Note that, in contrast to our previous study [18], where the same AO center axis was used for the manual and the SA method, the new method automatically estimates the AO axis. Thus, the $A O$ result was evaluated using the entire surface and the common region only (i.e. clipping both automatic and manual surfaces with the surface's limits). A paired $t$-test $(p<0.05)$ between the SA and FA errors when compared with manual contours was used to check for statistically significant differences in the results. Moreover, the error obtained for each stage of the FA error was also evaluated and compared with a paired t-test $(p<0.05)$. Finally, the computational time of the FA approach was registered. All results were computed using MATLAB code on an Intel (R) i7 CPU at $2.8 \mathrm{GHz}$ and $16 \mathrm{~GB}$ of RAM. A $\mathrm{C}++$ implementation of the competitive strategy and of the registration algorithm [31] was used.

Results: Table 1 shows the differences between the results obtained by the FA and the manual approaches. Of note, the FA was not feasible in two cases, namely: in one case the AO axis was wrongly initialized (entering inside the superior vena cava), and, in another one, the TV valve was wrongly estimated. Moreover, the SA results were also presented against the ones obtained by the FA version, showing a similar performance for the LA and RA segmentations. Again, the maximal accuracy was obtained for the LA chamber with an error of $1.05 \pm 0.19 \mathrm{~mm}$ (FA version). A notable improvement was observed for the AO (SA vs FA), achieving notstatistically significant differences only for the clipped version.

Fig. 5 presents the FA segmentation error in each stage of the proposed method, showing a significant reduction of the error between all steps. Finally, representative FA segmentation results are shown in Fig. 6. For clarity sake, we also presented the IAS wall contour (cyan contour) which is applied to estimate the FO region. Regarding the computational time, the contours initialization through an atlas-based technique took $61.4 \pm 5.1 \mathrm{~s}$, while the competitive segmentation required $42.1 \pm 6.0 \mathrm{~s}$. The manual analysis made by the expert took approximately 10 min per patient.

\subsection{Fully automatic identification of the fossa ovalis region}

Ground truth generation: Two experts (Obs. 1 and Obs. 2) identified the FO region in all the 41 datasets. Moreover, one expert repeated the analysis for 10 cases two weeks later. In detail, multiple $2 \mathrm{D}$ slices of the FO were delineated and then interpolated into a 3D surface. Manual corrections were finally performed to improve the result. The manual delineation strategy was performed using the MITK (Medical Imaging Interaction Toolkit, [32]).

Implementation details: Here, the IAS contour (Section 2.3.1) was defined using a spherical representation with a total of $40 \times 40$ points and the regional intensity-based energies were defined using image profiles with $3 \mathrm{~mm}$ inward and outward of the contour. The $F O_{\text {Spatial }}$ map was estimated using all manually evaluated CTs as temples using a leave-one-out strategy (i.e. the target case was removed from the database). The influence of the number of templates used is addressed in Supplementary Material III. Regarding the relative threshold (Section 2.3.3), the 90th quartile of the probabilistic map was used. It should be noted that the threshold was defined without the positions with probability equal to 0 . For further details about parameter tuning, the reader is kindly directed to the supplementary material III.

Statistical analysis: The FA results were compared with the corresponding manual contours in terms of P2S, Dice and centroid position error, i.e. computed as the distance between the centroid of both surfaces (CPE). The intra- and inter-observer variability was computed using the difference between the manual analysis, and compared with the FA results using an unpaired and paired $t$-test $(p<0.05)$, respectively. Finally, the computational time was registered and compared with the manual practice.

Results: The FA method was able to estimate successfully the FO in all the 41 cases. Overlaps between FA and manual surfaces were always obtained and the optimal FO position (maximum value of $F O_{\text {Position }}$ ) was always found inside the manual contours. Fig. 7 shows the intra- and inter-observer variability and also the evaluation results of the FA method against the manual analysis in terms of P2S, Dice and CPE. A similar performance was found between the FA method and inter-observer variability for the P2S (intra-observer: $1.39 \pm 0.35 \mathrm{~mm}$, inter-observer: $1.74 \pm 0.68 \mathrm{~mm}$, FA-Obs1: $1.77 \pm 0.66 \mathrm{~mm}$ and FA-Obs2: $1.88 \pm 0.58 \mathrm{~mm}$ ) and Dice (intra-observer: $70.66 \pm 4.74 \%$, inter-observer: $65.40 \pm 9.08 \%$, FAObs1: $60.92 \pm 11.92 \%$ and FA-Obs2: $61.33 \pm 9.06 \%)$. Statistically significant differences were found between the FA evaluation and the inter-observer variability for the CPE error (intraobserver: $2.50 \pm 1.37 \mathrm{~mm}$, inter-observer: $2.43 \pm 1.20 \mathrm{~mm}$, FA-Obs1: $2.90 \pm 1.28 \mathrm{~mm}$ and FA-Obs2: $2.90 \pm 1.17 \mathrm{~mm}$ ). Fig. 8 allows the assessment of these differences by showing the spatial distribution of the CPE. In detail, the error between the manual (mean of both observers) and the automatic centroid in each spatial direction (i.e. axial, sagittal and coronal) was computed. A clear tendency to generate the FO contour superiorly was found with the manual method when compared with the FA one. Fig. 9 shows representative FO results. Finally, the FA required $15.0 \pm 1.6,21.3 \pm 1.9 \mathrm{~s}$ for the spatial location map and $0.7 \mathrm{~s}$ for the algorithm's steps 2.3.1, 
Table 1

Point-to-surface (P2S) error, Dice coefficient and 95th percentile of Hausdorff distance obtained between the proposed method (semi-automatic - SA and fully automatic - FA) against manual contours for atrial region segmentation.

\begin{tabular}{llllllll}
\hline & & P2S $(\mathrm{mm})$ & & \multicolumn{2}{c}{ Dice } & \multicolumn{2}{c}{ 95th perc. Hausdorff (mm) } \\
Segmentation & SA & FA & SA & FA & SA & FA \\
\hline LA & Entire & $1.54 \pm 0.32$ & $1.52 \pm 0.33$ & $0.91 \pm 0.01$ & $0.91 \pm 0.01$ & $6.52 \pm 2.41$ & $6.64 \pm 1.75$ \\
& Cropped & $1.07 \pm 0.17$ & $1.05 \pm 0.19$ & $0.95 \pm 0.01$ & $0.95 \pm 0.01$ & $3.36 \pm 0.98$ & $3.38 \pm 0.80$ \\
RA & Entire & $2.13 \pm 0.51$ & $2.17 \pm 0.54$ & $0.87 \pm 0.03$ & $0.86 \pm 0.04$ & $8.13 \pm 2.18$ & $8.10 \pm 1.98$ \\
& Cropped & $1.68 \pm 0.47$ & $1.69 \pm 0.35$ & $0.91 \pm 0.02$ & $0.92 \pm 0.02$ & $6.03 \pm 2.04$ & $6.11 \pm 1.34$ \\
AO & Entire & $0.65 \pm 0.12^{*}$ & $0.83 \pm 0.44^{*}$ & $0.94 \pm 0.02^{*}$ & $0.91 \pm 0.05^{*}$ & $1.61 \pm 0.33^{*}$ & $2.28 \pm 1.71^{*}$ \\
& Clipped & - & $0.71 \pm 0.38$ & - & $0.94 \pm 0.04$ & - & $1.87 \pm 1.46$ \\
\hline
\end{tabular}

*paired $t$-test between the FA and SA result $(p<0.05)$. LA - left atrium, RA - right atrium, AO - aortic tract.

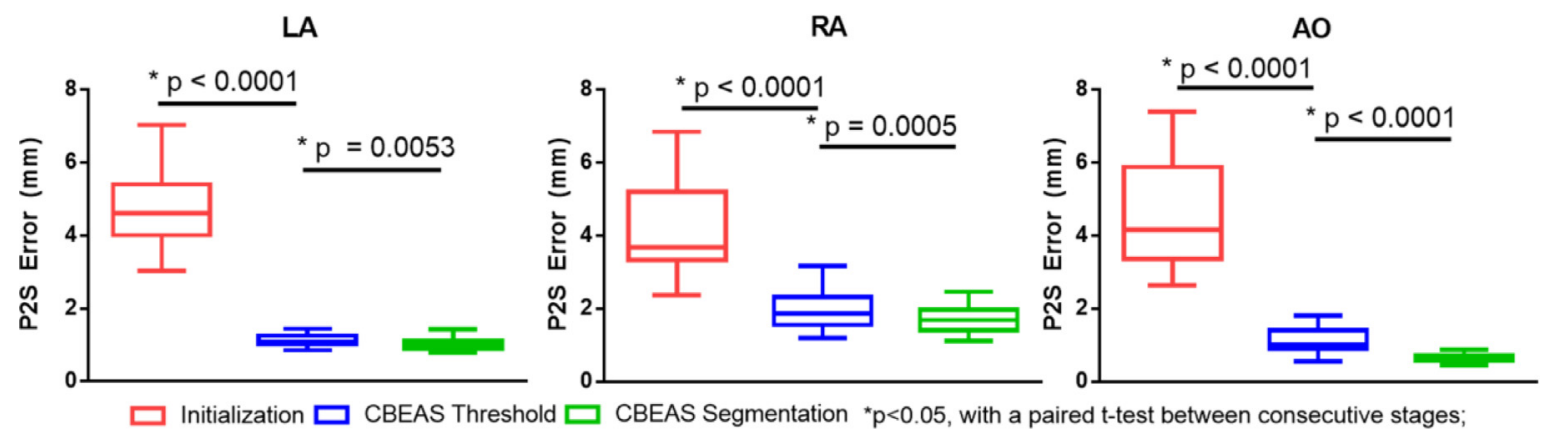

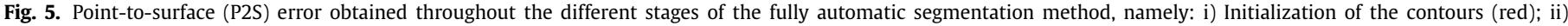

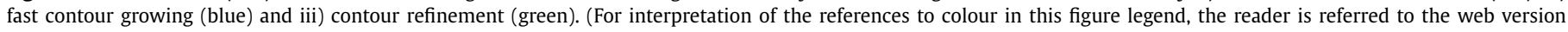
of this article.)

2.3.2, and 2.3.3, respectively. The manual delineation took approximately 4 min per patient.

\section{Discussion}

In the article, we present a novel fully automatic strategy to segment both atrial region models and the fossa ovalis. The proposed method is divided into three sequential stages with a robust initialization through the use of an atlas, atrial chambers segmentation and mid-walls using a competitive approach and anatomical landmark identification by combining wall thickness information and spatial landmark location. Globally, the proposed framework showed a high feasibility and accuracy, corroborating its addedvalue for IAS intervention planning. Although in two independent cases the segmentation was considerable not feasible, possibly requiring a small user input correction, acceptable results were achieved for all the FO surfaces. Note that, no user-input was allowed and all results were obtained without any user interaction. Indeed, since an accurate delineation of the atrial and aortic walls was still achieved in these two cases, correct identification of the FO was possible.

The importance of a correct IAS intervention planning through identification of relevant landmarks was previously described $[9,22,23,33,34]$. In addition to be crucial in abnormal anatomical situations, correct planning also improves the physician's confidence, reduces the number of procedural failures, eases the entire intervention, and improves the intervention result $[5,9,33]$. Nevertheless, the planning stage is usually performed manually $[9,22]$, which is extremely time-consuming (taking more than 14 min per patient) and presents high intra-/inter-observer variability, hampering its application during clinical routine. In this sense, the proposed FA strategy appears as a potential and promising solution, showing high robustness, accuracy and low computational burden (full planning requires $2-3 \mathrm{~min}$ ). Regarding its computational cost and taking into consideration the expected high variability of the FOV used in clinical CT acquisitions (i.e. cardiac region only, full thorax, full body, among others), different strategies were implemented to reduce the influence of the number of samples used (i.e. image size) in the computational burden of the proposed method, namely: 1) pre-processing of the entire image to detect a target FOV throughout the atlas alignment stage; 2 ) competitive segmentation using regional-based energies defined based on the contour's position; and 3) FO identification totally based on 3D surfaces only. To the author's best knowledge, no previous work focusing on the automatic planning of IAS interventions was presented, being a clear novelty of this work. Currently, few software tools are available to evaluate all cardiac chambers and even great vessels $[14,15]$. However, evaluation of the atrial/aortic walls is not possible $[14,17]$. Finally, and in a different way, some researchers are now exploring the potentialities of novel augmented reality setups, fusing multiple imaging modalities, to ease the visual identification of the FO [21].

Starting to evaluate the proposed segmentation method, the combination of both global atlas alignment with a refinement through a competitive strategy showed its high accuracy and feasibility (Table 1 ), with a performance comparable to other automatic methods [15] $[14,16]$. Additionally to the described clinical database, the method's accuracy was also corroborated in one recent benchmark (STACOM 2013 [2], supplementary material IV), allowing a direct comparison between the errors obtained by the FA method and the variability between manual observers, which was not possible by the clinical database used. Similarly to our previous study [18], the RA showed the highest errors (approximately $1.7 \mathrm{~mm}$ for the FA and SA), which is explained by the noisy regions found, the difficulty in distinguishing between $\mathrm{VC}$ and atrial body, and even low contrast or missing walls at the TV valve plane. No differences were achieved between the SA and FA methods for the LA and RA results. A similar performance between FA and SA methods was observed for the entire and cropped contours situation (Table 1 and Fig. 6). In contrast, significant differences were found between the SA and FA for the entire AO contour, due to the initialization used in the new method (Table 1). While both SA and 

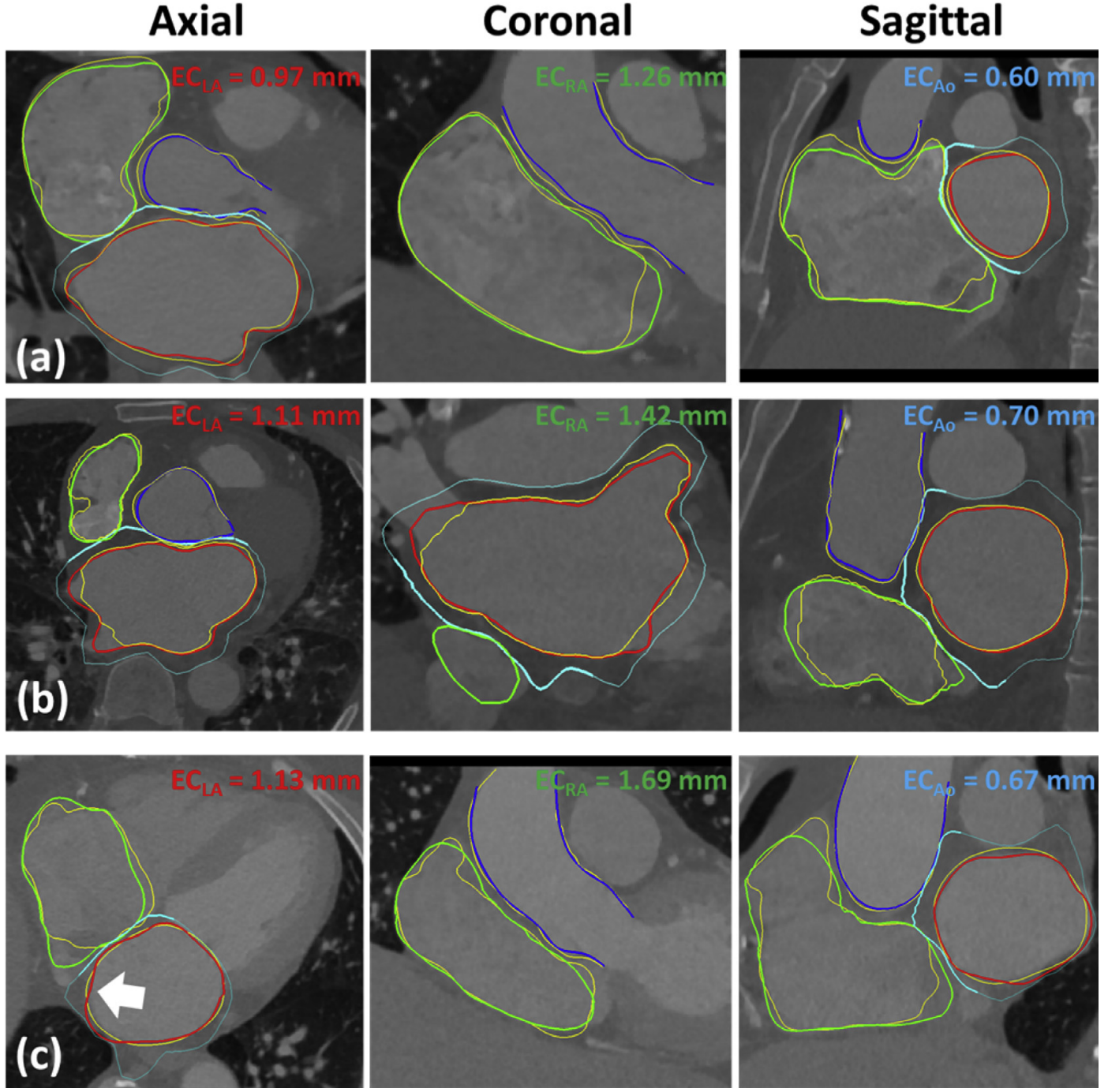

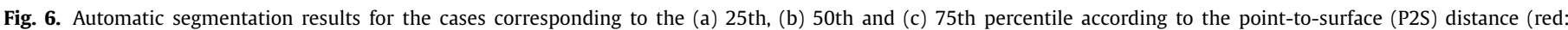

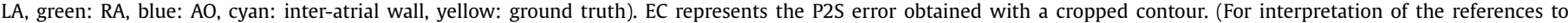
colour in this figure legend, the reader is referred to the web version of this article.)

manual contouring were performed using the same centerline, the FA estimates the AO axis totally based on the image content (coronal view of Fig. 6A). As such and as previously observed in other studies [13,35], small differences between central axis will result in high P2S errors. When evaluating the common AO region, i.e. clipped version, no statistically significant differences were found, proving that the lower performance found for the FA is related to the initialization strategy applied. Regarding the computational time, and similar to the previous observed in [18], the segmentation through a competitive based BEAS approach corroborated its low computational time (less than $1 \mathrm{~min}$. to segment the LA, RA and $\mathrm{AO}$ ) and attractiveness for normal clinical practice.

Similarly to the multi-atlas segmentation strategies [16,17], the high feasibility of the proposed method is related with the atlasbased technique applied and its atlas database. Only a rough estimation of each chamber (by identifying its central region) is required by our methodology, being posteriorly refined to the real anatomy based on the spatial location of the multiple contours (Fig. 5). As such, the result of the proposed method is theoretically not so sensitive to the atrial anatomical shape variability available in the atlas database, as observed for the totally-based multi-atlas case. In fact, our methodology appears to be more versatile for atrial region segmentation when compared with pure atlas-based techniques, particularly in abnormal cases. Note that the clinical database was constructed by retrospectively and sequentially selecting datasets from the hospital database. Therefore, a high shape variability and even different image properties are found between the different subjects, including normal and multiple pathological cases (supplementary material II). Regarding the computational time, the proposed method is much faster (approximately $2 \mathrm{~min}$ in a common personal computer laptop) than traditional atlas approaches, making it more attractive for clinical practice. However, the authors would like to mention that the multi-atlas strategies are generic formulations with a large application in different scenarios (not only the heart) [24] and without any model description limitation [16,17]. Moreover, although we considered our method to be fast, optimized tools that segment all chambers in a few seconds are available [14,15].

In a second stage, we presented a novel strategy to extract relevant landmarks from the aforementioned atrial models. Since the automatic FO detection stage is totally dependent of the initial contours, the current FO strategy is not feasible without an accurate segmentation process. Contrary to previous studies where the FO is visually detected $[34,36]$, either by manually marking on one specific image position [37], or by identifying the thinnest position of the atrial wall in two/three orthogonal planes [22], we automat- 


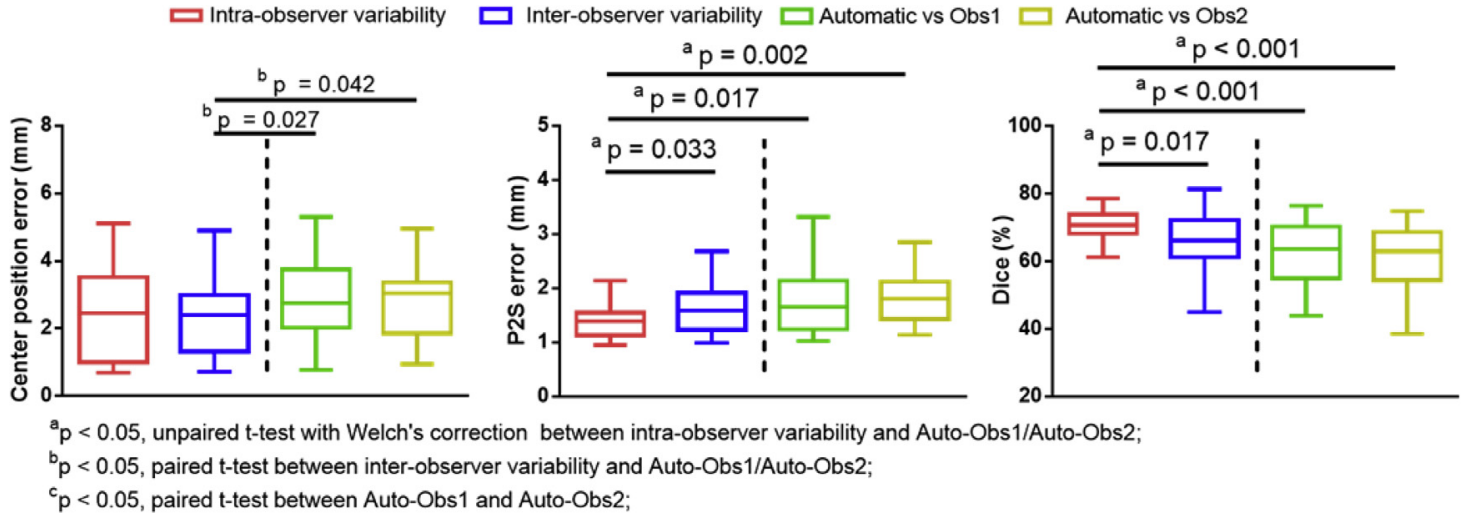

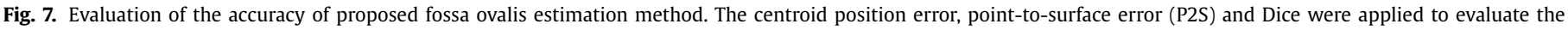
fully automatic (FA) method against two manual observers (Obs1, Obs2). The intra- and inter-observer variability is also presented.

ically extract an entire 3D surface showing the extent of the FO region. This realistic surface can be used to facilitate and improve the detection of the optimal access routes for transseptal puncture by identifying the optimal position that guarantees maximum catheter dexterity at the left side $[9,23,36,38]$, as a relevant landmark region for IAS defect closure [39] and for optimal device selection for other LA procedures $[6,20,21]$. In order to identify the FO, we combined both patient-specific information, measured as the IAS wall thickness and spatial maps representing the expected FO position. The IAS wall thickness is defined based on the initial atrial contours, being used as fixed references. Although the FO contour is defined along the LA surface, only the points at the interaction positions (and its neighbors, dark cyan line in Fig. 6) between the different contours are relevant for the identification of the FO. In order to obtain a high detail on IAS wall, low smoothing terms were applied, making it correctly defined for the IAS region but a little jaggy for the remaining regions.

Regarding the FO spatial map strategy, it aligns the unknown case with a set of different anatomies, transferring the labeled FO for the novel patient. Although such strategy makes the results dependent on the anatomies available in the template database, it only generates a global map describing the anatomical regions where the FO can be found. Indeed, the influence of the number of cases used on the template database was also evaluated, obtaining a similar performance when using half or the entire database, corroborating therefore the method's robustness (supplementary material III). It might be noted that the computational cost of this step is directly related to the number of templates used. Nevertheless, since we only need to estimate a global map of the FO location, a high number of templates is not required, thus keeping the method computationally attractive. Then, and in order to adapt the result to the particularities of each patient, the global map is refined using the patient-specific anatomy extracted from the wall thickness map. In fact, accurate detection of the FO region using only the spatial or thickness map is not feasible (supplementary material III). Therefore, the combination of both IAS wall thickness and the spatial maps is required to compensate for possible segmentation errors, generating a probabilistic map that represents the optimal FO positions. Moreover, the application of one spatial map is also crucial to make the current strategy less sensitive to the image acquisition parameters (e.g. differences in terms of contrast). As a final remark, although the obtained probabilistic map could be directly used as a relevant reference for interventional planning, we decided to apply a fixed threshold to binarize the most probable region. Of note, no over-tuning of this parameter was not applied and a different value could be also used without losing performance. Different threshold strategies with extra terms (such as area, maximum length, adaptive approach) could be an interesting solution to improve the method's performance. Nevertheless, we would like to emphasize that the maximum probability's position was included inside the manual FO surfaces (by both observers), corroborating the method's accuracy.

Fig. 7 allows one to conclude that the FA method presented a performance similar to the inter-observer variability for the FO identification, validating the potential of the proposed method. Statistically significant differences were found between the intra-observer variability and the remaining cases, which can be explained by the small number of cases used and even by different manual delineation strategies applied. While one of the observers tended to segment the inner portion of the central depression of the FO only, the second one included both FO and the initial transition of the FO limbus, obtaining a larger contour. Interestingly, our automatic strategy typically generates a contour at the intermediate position of both manual contours (Fig. 9). The CPE results corroborated the high accuracy of the proposed automatic method, particularly, considering the mean size - $17 \pm 2.7 \mathrm{~mm}$ of the FO in the test database, but with statistically significant differences against the inter-observer variability, which indicates a misalignment between manual and FA surfaces. These differences can be explained by the sub-optimal segmentation result at the top of the atrial roof, due to its high curvature and its irregular shape (white arrow on Fig. 6C and Fig. 9E). These last observations are corroborated by Fig. 8, where it is depicted that the manual centroid positions are typically located superiorly (at the coronal and sagittal directions) than the centroid of the automatic result.

The authors would like to emphasize that although the proposed methodology, focused on anatomical alignment and contour refinement, proved its high accuracy and robustness for the segmentation of the atrial region (mainly at the thin mid atrial walls) and fossa ovalis, different solutions based on machine learning approaches (principally deep learning techniques) are also potentially interesting. Indeed, such solutions are expected to be developed during the next years, both for the segmentation of the cardiac chambers and even for the fossa ovalis region. Nevertheless, when comparing end-to-end deep learning approaches with the proposed technique, there are several challenges that need to be addressed in the near future: 1) to the author's best knowledge, large training databases or even benchmarks with strong manual references delineated by multiple observers are not available for this problem; 2) due to its low thickness (in some situations could be one pixel or even not visible), correct modelling of the interatrial is not straightforward with the pure machine learning approach, requiring further research; and 3) method's sensitivity to 

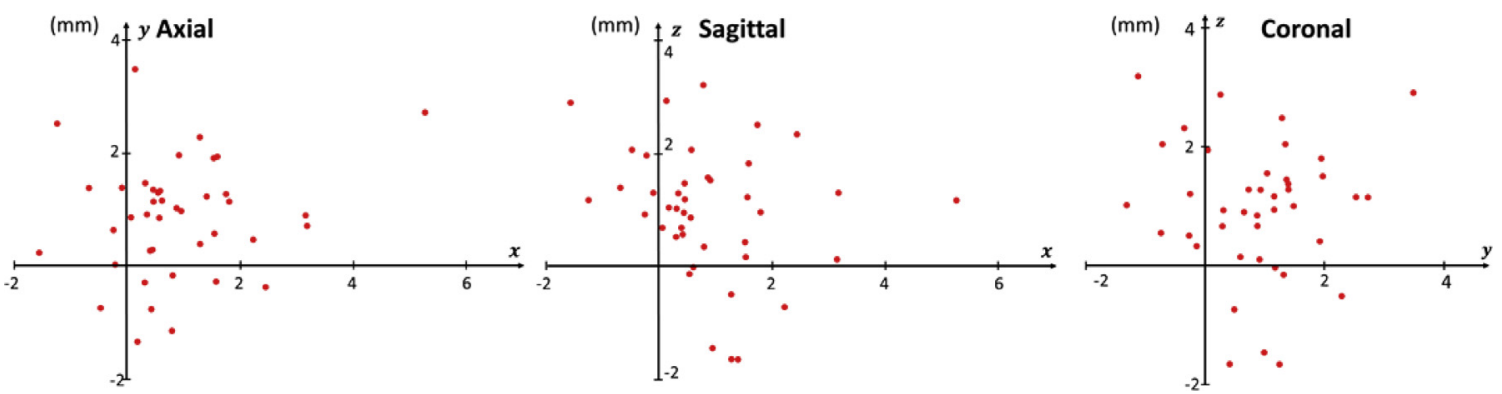

Fig. 8. Distribution of the spatial centroid position errors (axial, coronal and sagittal directions) between the mean manual results and the automatic ones.

Axial
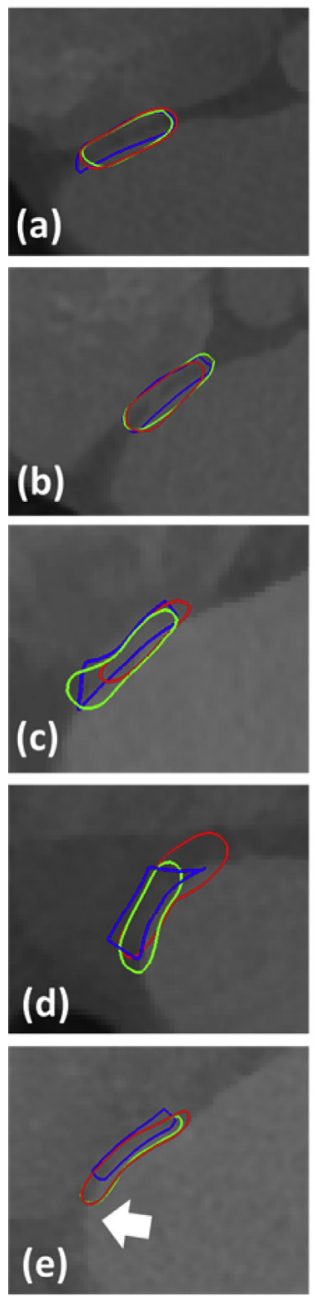

Coronal
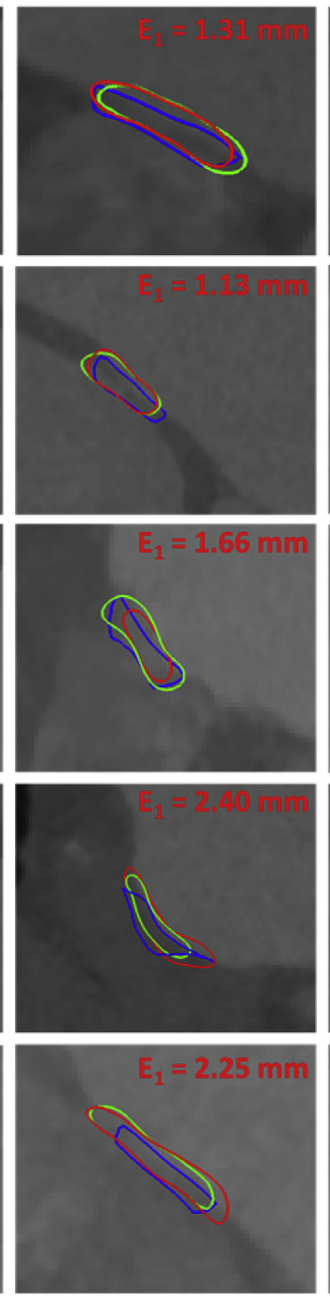

Sagittal
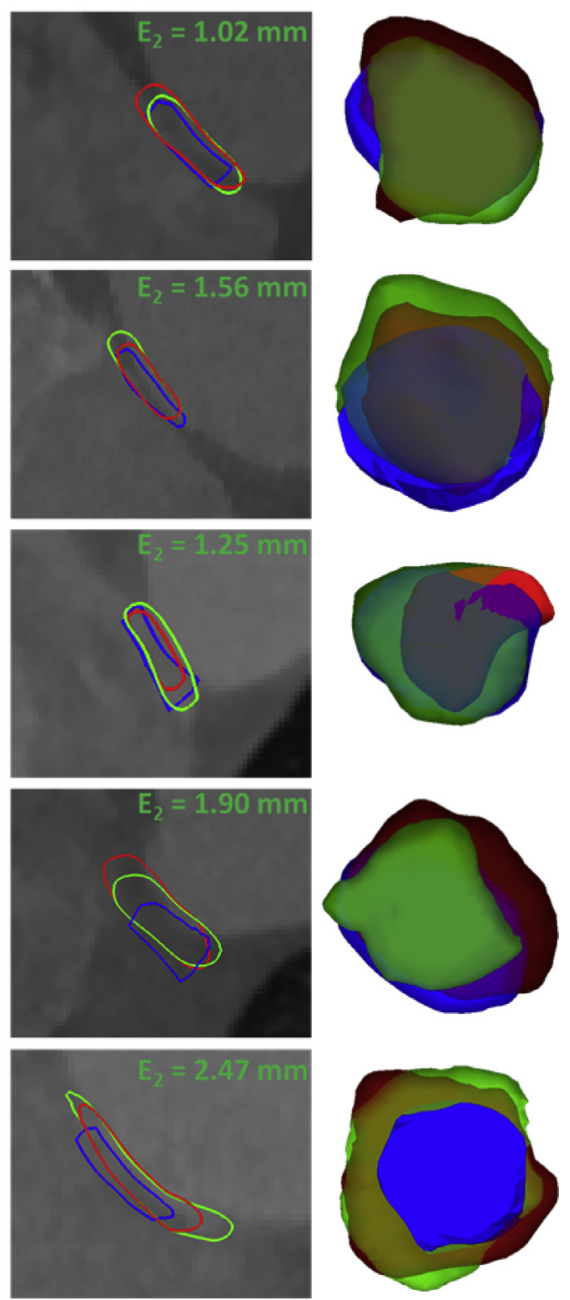

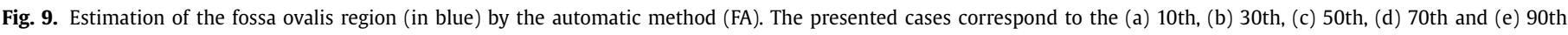

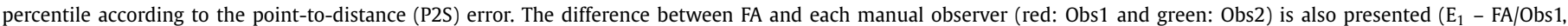
$\mathrm{E}_{2}-\mathrm{FA} / \mathrm{Obs} 2$ ). (For interpretation of the references to colour in this figure legend, the reader is referred to the web version of this article.)

image artifacts (e.g. pacemakers) or abnormal anatomies (possibly not represented in the training database) needs to be studied.

As a future work, we intend to apply the proposed framework for real planning of inter-atrial intervention [9,22]. Not only the physician will use the automatically estimated surfaces to identify the optimal path, as also the obtained models will be included in the real intervention (combining fluoroscopy and CT data through [9]), improving the interventional data and easing the identification of relevant anatomical regions. The FO will be represented as an accurate 3D surface, representing its entire shape and not only one specific point [37], easing the selection of the optimal route and preventing procedural complications/failures [5,6]. Furthermore, we also believe that our method could also be applied to improve IAS intervention simulation tools [23], by applying realistic and accurate models of the atrial region and the FO, instead of mean atrial region models or entire IAS wall surfaces. Finally, the proposed fully-automatic atrial region segmentation strategy can be efficiently applied in clinical practice for accurate evaluation of the relevant global indicators (e.g., volume, dimension) of the LA, RA and AO. Moreover, although it was not within the scope of the current study, the current fully-automatic methodology can be extended, keeping its high accuracy and robustness, for 
other cardiac chambers (e.g. LV) or even different imaging modalities (e.g. 3D magnetic resonance imaging, requiring only a different atlas database), thereby increasing the potential of the proposed framework for the clinical environment. Nevertheless, and as explained in our previous study [18], segmentation of the RV with the current atlas-based approach followed by competitive BEAS is not feasible. Since BEAS relies on explicit functions, intrinsic shape limitations are imposed (e.g. spherical or cylindrical space), being therefore - in its present form - not suitable to describe complex anatomies as the RV.

The current study has some limitations, namely: 1) accurate identification of FO is unfeasible on non-contrasted CT or datasets with larger pixel spacing due to the low contrast and resolution of the thin IAS wall (as a consequence, a non-refined $I A S_{\text {wall }}$ map is obtained), respectively; 2) extreme abnormal atrial anatomies can present a sub-optimal result when performing the atlas alignment or when estimating the $\mathrm{FO}_{\text {Spatial }}$ map due to the limited shape representation on the reference templates; 3 ) a sub-optimal FO result can be obtained in patients with patent foramen ovale; and 4) a larger and multi-center database with manual references from several experts is required to exhaustive validate the proposed pipeline.

\section{Conclusions}

The proposed fully automatic framework showed its promising for IAS interventional planning, making the planning more accurate, simpler, without observer variability and faster. The new atrial region segmentation approach obtained results comparable to the remaining state-of-the-art methods, but providing superior results for the mid atrial walls. No differences were found between the new and our previous segmentation approaches; however, it should be noted that with the new approach, the segmentation is free of user interaction. Regarding the fossa ovalis segmentation, the proposed method obtained results similar to the ones manually delineated by experts, allowing a correct and fast identification of the optimal route for transseptal puncture or optimal device selection for LA interventions.

\section{Acknowledgements}

The authors acknowledge Fundacão para a Ciência e a Tecnologia (FCT), in Portugal, and the European Social Found, European Union, for funding support through the "Programa Operacional Capital Humano" (POCH) in the scope of the $\mathrm{PhD}$ grants SFRH/BD/95438/2013 (P. Morais) and SFRH/BD/93443/2013 (S. Queirós)

This work was funded by projects NORTE-01-0145-FEDER000013, NORTE-01-0145-FEDER-000022 and NORTE-01-0145FEDER-024300, supported by Northern Portugal Regional Operational Programme (Norte2020), under the Portugal 2020 Partnership Agreement, through the European Regional Development Fund (FEDER), and also been funded by FEDER funds, through Competitiveness Factors Operational Programme (COMPETE), and by national funds, through the FCT, under the scope of the project POCI-01-0145-FEDER-007038.

\section{Conflict of interest}

The authors declare that they have no conflict of interest.

\section{Supplementary materials}

Supplementary material associated with this article can be found, in the online version, at doi:10.1016/j.cmpb.2018.04.014.

\section{References}

[1] R.H. Anderson, R. Razavi, A.M. Taylor, Cardiac anatomy revisited, J. Anat. 205 (2004) 159-177.

2] C. Tobon-Gomez, A.J. Geers, J. Peters, J. Weese, K. Pinto, R. Karim, M. Ammar, A. Daoudi, J. Margeta, Z. Sandoval, Benchmark for algorithms segmenting the left atrium from 3D CT and MRI datasets, IEEE Trans. Med. Imaging 34 (2015) $1460-1473$.

[3] T.H. Hauser, D.C. Peters, J.V. Wylie, W.J. Manning, Evaluating the left atrium by magnetic resonance imaging, Europace 10 (2008) iii22-iii27.

[4] W.P. Abhayaratna, J.B. Seward, C.P. Appleton, P.S. Douglas, J.K. Oh, A.J. Tajik, T.S. Tsang, Left atrial size: physiologic determinants and clinical applications, J. Am. Coll. Cardiol. 47 (2006) 2357-2363.

[5] P. Morais, J.L. Vilaça, J. Ector, J. D’hooge, J.M.R. Tavares, Novel solutions applied in transseptal puncture: a systematic review, J. Med. Devices 11 (2017) 010801.

[6] V.C. Babaliaros, J.T. Green, S. Lerakis, M. Lloyd, P.C. Block, Emerging applications for transseptal left heart catheterizationold techniques for new procedures, J. Am. Coll. Cardiol. 51 (2008) 2116-2122.

[7] K. Cleary, T.M. Peters, Image-guided interventions: technology review and clinical applications, Annu. Rev. Biomed. Eng. 12 (2010) 119-142.

[8] Y. Yao, L. Ding, W. Chen, J. Guo, J. Bao, R. Shi, W. Huang, S. Zhang, T. Wong, The training and learning process of transseptal puncture using a modified technique, Europace (2013) eut078

[9] F. Bourier, T. Reents, S. AMMAR-BUSCH, V. Semmler, M. Telishevska, M. Kottmaier, C. Lennerz, C. Grebmer, C. Kolb, I. Deisenhofer, Transseptal puncture guided by CT-derived 3D-augmented fluoroscopy, J. Cardiovasc. Electrophysiol. (2016)

[10] R. Liao, S. Miao, Y. Zheng, Automatic and efficient contrast-based 2-D/3-D fusion for trans-catheter aortic valve implantation (TAVI), Comput. Med. Imaging Graphics 37 (2013) 150-161.

[11] J.J. Thaden, S. Sanon, J.B. Geske, M.F. Eleid, N. Nijhof, J.F. Malouf, C.S. Rihal, C.J. Bruce, Echocardiographic and fluoroscopic fusion imaging for procedural guidance: an overview and early clinical experience, J. Am. Soc. Echocardiogr. 29 (2016) 503-512

[12] X. Huang, J. Moore, G. Guiraudon, D.L. Jones, D. Bainbridge, J. Ren, T.M. Peters, Dynamic 2D ultrasound and 3D CT image registration of the beating heart, IEEE Trans. Med. Imaging 28 (2009) 1179-1189.

[13] S. Queiros, A. Papachristidis, P. Morais, K.C. Theodoropoulos, J.C. Fonseca, M.J. Monaghan, J.L. Vilaca, J. D'hooge, Fully automatic 3D-TEE segmentation for the planning of transcatheter aortic valve implantation, IEEE Trans. Biomed. Eng. 64 (2016) 1711-1720.

[14] O. Ecabert, J. Peters, M.J. Walker, T. Ivanc, C. Lorenz, J. von Berg, J. Lessick, M. Vembar, J. Weese, Segmentation of the heart and great vessels in CT images using a model-based adaptation framework, Med. Image Anal. 15 (2011) $863-876$

[15] Y. Zheng, A. Barbu, B. Georgescu, M. Scheuering, D. Comaniciu, Four-chamber heart modeling and automatic segmentation for 3-D cardiac CT volumes using marginal space learning and steerable features, IEEE Trans.on Med. Imaging 27 (2008) 1668-1681.

[16] H. Kirişli, M. Schaap, S. Klein, S. Papadopoulou, M. Bonardi, C.-H. Chen, A. Weustink, N. Mollet, E. Vonken, R. van der Geest, Evaluation of a multi-atlas based method for segmentation of cardiac CTA data: a large-scale, multicenter, and multivendor study, Med, Phys. 37 (2010) 6279-6291.

[17] M.A. Zuluaga, M.J. Cardoso, M. Modat, S. Ourselin, Multi-atlas propagation whole heart segmentation from MRI and CTA using a local normalised correlation coefficient criterion, in: Functional Imaging and Modeling of the Heart, Springer, 2013, pp. 174-181.

[18] P. Morais, J.L. Vilaça, S. Queirós, F. Bourier, I. Deisenhofer, J.M.R.S. Tavares, J. D'Hooge, A competitive strategy for atrial and aortic tract segmentation based on deformable models, Med. Image Anal. 42 (2017) 102-116.

[19] R. Shahzad, D. Bos, R.P. Budde, K. Pellikaan, W.J. Niessen, A. van der Lugt, T. van Walsum, Automatic segmentation and quantification of the cardiac structures from non-contrast-enhanced cardiac CT scans, Phys. Med. Biol. 62 (2017) 3798.

[20] Y. Wang, L. Di Biase, R.P. Horton, T. Nguyen, P. Morhanty, A. Natale, Left atrial appendage studied by computed tomography to help planning for appendage closure device placement, J. Cardiovasc. Electrophysiol. 21 (2010) 973-982.

[21] F.F. Faletra, L. Biasco, G. Pedrazzini, M. Moccetti, E. Pasotti, L.A. Leo, G. Cautilli, T. Moccetti, M.J. Monaghan, Echocardiographic-fluoroscopic fusion imaging in transseptal puncture: a new technology for an old procedure, J. Am. Soc. Echocardiogr. 30 (2017) 886-895.

[22] S. Verma, S. Adler, A. Berman, A. Duran, D. Loar, Localization of fossa ovalis and Brockenbrough needle prior to left atrial ablation using three-dimensional mapping with EnSite fusion ${ }^{\mathrm{TM}}$, J. Interv. Card. Electrophysiol. 30 (2011) 37-44.

[23] J. Jayender, R.V. Patel, G.F. Michaud, N. Hata, Optimal transseptal puncture location for robot-assisted left atrial catheter ablation, Int. J. Med. Robotics Comput. Assist. Surg. 7 (2011) 193-201.

[24] J.E. Iglesias, M.R. Sabuncu, Multi-atlas segmentation of biomedical images: a survey, Med. Image Anal. 24 (2015) 205-219.

[25] N. Otsu, A threshold selection method from gray-level histograms, Automatica 11 (1975) 23-27.

[26] S. Queirós, D. Barbosa, B. Heyde, P. Morais, J.L. Vilaça, D. Friboulet, O. Bernard, J. D'hooge, Fast automatic myocardial segmentation in 4D cine CMR datasets, Med. Image Anal. 18 (2014) 1115-1131.

[27] D. Barbosa, T. Dietenbeck, J. Schaerer, J. D’hooge, D. Friboulet, O. Bernard, B-spline explicit active surfaces: an efficient framework for real-time 3-D region-based segmentation, IEEE Trans. Image Process. 21 (2012) 241-251. 
[28] J. Pedrosa, D. Barbosa, B. Heyde, F. Schnell, A. Rösner, P. Claus, J. D’hooge, Left ventricular myocardial segmentation in 3-D ultrasound recordings: effect of different endocardial and epicardial coupling strategies, IEEE Trans. Ultrason. Ferroelectr. Freq. Control 64 (2017) 525-536.

[29] B. Amberg, S. Romdhani, T. Vetter, Optimal step nonrigid icp algorithms for surface registration, computer vision and pattern recognition, 2007, in: IEEE Conference on CVPR'07, IEEE, 2007, pp. 1-8

[30] A.H. Squillacote, J. Ahrens, The Paraview Guide, Kitware, 2007.

[31] S. Klein, M. Staring, K. Murphy, M.A. Viergever, J.P. Pluim, Elastix: a toolbox for intensity-based medical image registration, IEEE Trans. Med. Imaging 29 (2010) 196-205.

[32] I. Wolf, M. Vetter, I. Wegner, T. Böttger, M. Nolden, M. Schöbinger, M. Hastenteufel, T. Kunert, H.-P. Meinzer, The medical imaging interaction toolkit, Med. Image Anal. 9 (2005) 594-604.

[33] P. Wagdi, H. Alkadhi, Can computer tomography help predict feasibility of transseptal puncture after percutaneous closure of an interatrial septal communication? J. Interv. Card. Electrophysiol. 34 (2012) 167-172.

[34] S. Knecht, H. Skali, M.D. O'neill, M. Wright, S. Matsuo, G.M. Chaudhry, C.I. Haffajee, I. Nault, G.H. Gijsbers, F. Sacher, Computed tomography-fluoroscopy overlay evaluation during catheter ablation of left atrial arrhythmia, Europace 10 (2008) 931-938.
[35] S. Queirós, C. Dubois, P. Morais, T. Adriaenssens, J.C. Fonseca, J.L. Vilaça, J. D’hooge, Automatic 3D aortic annulus sizing by computed tomography in the planning of transcatheter aortic valve implantation, J. Cardiovasc. Comput. Tomogr. (2016).

[36] S. Knecht, I. Nault, M. Wright, S. Matsuo, N. Lellouche, P.E. Somasundaram, M.D. O'neill, K.-T. Lim, F. Sacher, A. Deplagne, Imaging in catheter ablation for atrial fibrillation: enhancing the clinician's view, Europace 10 (2008) iii2-iii7.

[37] L.N. Graham, I.C. Melton, S. MacDonald, I.G. Crozier, Value of CT localization of the fossa ovalis prior to transseptal left heart catheterization for left atrial ablation, Europace 9 (2007) 417-423.

[38] D.R. Tomlinson, N. Sabharwal, Y. Bashir, T.R. Betts, Interatrial septum thickness and difficulty with transseptal puncture during redo catheter ablation of atrial fibrillation, Pacing Clin. Electrophysiol. 31 (2008) 1606-1611.

[39] F.F. Faletra, G. Perk, N.G. Pandian, H.-J. Nesser, I. Kronzon, Closure of patent foramen ovalis and atrial septal defect, in: Real-Time 3D Interventional Echocardiography, Springer, 2014, pp. 21-41. 